\title{
EFFECT OF DESIGN AND SURFACE TREATMENTS ON FRACTURE RE- SISTANCE OF ZIRCONIA INLAY-RETAINED FIXED DENTAL PROSTHESIS AFTER THERMO-MECHANICAL CYCLING: AN IN VITRO STUDY
}

\author{
Mohamed S Hussein*, Hussein R Mohammed ${ }^{* *}$, Hesham I Othman ${ }^{* * *}$
}

\begin{abstract}
Objective: This study evaluated the effect of preparation designs and surface treatment on the fracture resistance of zirconia inlay retained fixed dental prosthesis (IR-FDPs). Material and Methods: Twenty secondary premolars and twenty secondary molars were used in this study. Twenty zirconia IR-FDPs were constructed, divided into two main groups, ten specimens each, according to abutment preparation (box \& tube shape), each main group was subdivided into two sub groups according to surface treatment (Air abrasion \& MDP primer surface treatment). The IR-FDPs were cemented using dual cure resin cement, then the specimens were subjected to thermo-mechanical cyclic loading (TMCL). The fracture resistance was measured. Results: The results showed that there was no statistically significant difference between air abrasion and MDP for both designs (box \& tube shape). The effect of design showed that there was statistically significant difference for both surface treatments between tube and box design where box design showed higher fracture resistance than tube design for both surface treatments. Conclusion: Inlay retained fixed dental prosthesis (IR-FDPs) with box shape provide more fracture resistance than with tube shape. The air abrasion surface treatment shows similar fracture as the MDP primer surface treatment.
\end{abstract}

KEYWORDS: CAD /CAM, Inlay retained fixed dental prosthesis, monolithic zirconia, MDP primer

\section{INTRODUCTION}

Various methods have been used to restore a single missing tooth, including dental implants, inlay-retained FDP (IR-FDPs), traditional full coverage fixed dental prosthesis have been used to treat a single missing tooth based on the least invasive approach, whereby intact tooth conserved as much as possible ${ }^{(1)}$. Implant-supported FDPs are highly qualified alternatives to tooth-supported FDPs, patients often refuse this option due to their surgical intervention ${ }^{(2)}$. An inlay-retained FDP (IRFDPs ) is, less-invasive treatment modality and a more conservative option for restoration of damaged teeth, because it requires minimal tooth reduction ${ }^{(3)}$.

An important area of interest is the choice of the restorative material to optimize the performance of such restoration. Computer aided design /Computer aided manufacturing (CAD/CAM) technology allowed recently a noticed expansion in the range of materials available for dental use ${ }^{(4)}$. Monolithic zirconia, which in comparison to glass ceramic and porcelain veneered zirconia ceramic restorations are high strength, a more conservative preparation of teeth, great precision and complete computer aided manufacturing (5).

Debonding is reported as one of the most common technical complications for zirconia-based restorations and they still need a more reliable and long-lasting adhesion to the substrates ${ }^{(6)}$. Therefore, bonding to zirconia-based ceramics remains a

\footnotetext{
* Dentist B.D.S, Faculty of Dental Medicine, Sinai University

** Lecturer of Crown and bridge, Faculty of Dental Medicine for Boys, Al-Azhar University.

*** Professor of Crown and bridge, Faculty of Dental Medicine for Boys, Al-Azhar University.
} 
challenge because of its nonreactive surface which requires different techniques than the conventional ones used for preparing silica-based ceramics, i.e. hydrofluoric acid etching and silane coupling agent. Therefore, air-abrasion and functional monomers are common methods that used to enhance bonding of zirconia-based ceramic, consequently enhance marginal adaptation ${ }^{6,7)}$.

Mechanical failure of dental restorations occurs after many years in service, indicating a fatigue failure rather than acute overload ${ }^{(8)}$. Damage accumulates from cyclic contacts between maxillary and mandibular teeth and finally limits the survival probability and lifetime of the restorations ${ }^{(9)}$. Mechanical cyclic loading tests are aimed at stimulating the conditions of mastication in the oral cavity by inducing alternate stresses in the specimens thus reflects the behavior of restoration under function ${ }^{(8)}$.

The purpose of this study was to investigate and compare different design and surface treatment of monolithic zirconia IR-FPDs as regard the fracture resistance. Therefore; The hypothesis of this study was that the preparation design and the surface treatment will influence the fracture resistance of the monolithic zirconia IR-FDPs after thermomechanical cyclic loading (TMCL).

\section{MATERIAL AND METHODS}

Twenty zirconia IR-FDPs were constructed in this study. Specimens were divided into two main groups, ten specimens each, according to abutment preparation (box \& tub shape). Then each main group was subdivided into two sub groups according to surface treatment (Air abrasion \& MDP primer surface treatment), (Figure 1).

Forty freshly extracted intact and caries-free human teeth of similar size (20 mandibular $2^{\text {nd }}$ molars) and (20 mandibular $2^{\text {nd }}$ premolars) were collected. The teeth were cleaned by curettage and stored in a saline solution at room temperature. The roots of the teeth were covered with a (0.3-0.5) mmthick layer of poly vinyl acetate material (EasyVac Gasket, 3A MEDES) to simulate the physiological tooth movement using vacuum forming machine.

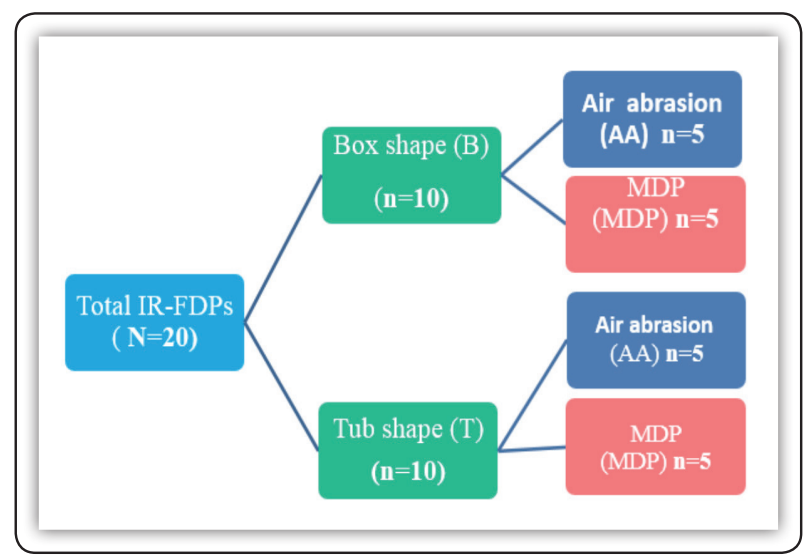

FIG (1) Diagram study design

A special machine milled aluminum holder was designed and fabricated to mount the abutment teeth inside acrylic block (Acrostone, Acrostone Dental Manufacture, Egypt) at $10 \mathrm{~mm}$ apart from each other representing the mesio-distal dimension of first mandibular molar ${ }^{[10-12]}$. The parallometer device (Paraflex, BEGO-Germany) was used to allow accurate vertical centralization of the tooth in the holder

The teeth were prepared with two different cavity preparation designs (box and tub shapes) according to Thompson et al ${ }^{[13]}$ general guidelines of inlay preparation using 5 axis $\mathrm{CNC}$ machines (CINCINNATI Milacron VT440-41). Box-shape dimension: in the molar abutment the occlusal part preparation was $\{4 \mathrm{~mm}$ wide (Bucco-lingually), $6 \mathrm{~mm}$ long (mesio-distally), and $2 \mathrm{~mm}$ deep\}, the proximal part extending $\{2 \mathrm{~mm}$ apical to the isthmus and floor was $1 \mathrm{~mm}$ wide (gingival seat)\}.

In the Premolar abutment \{the occlusal part was $3 \mathrm{~mm}$ wide (Bucco-lingually), $4 \mathrm{~mm}$ long (mesiodistally), and $2 \mathrm{~mm}$ deep\}, and the proximal part 
extending $\{2 \mathrm{~mm}$ apical to the isthmus and floor was $1 \mathrm{~mm}$ wide (gingival seat)\}. Tube shape dimension: The tub-shaped preparation consists of the occlusal part only which prepared with the same dimensions of the occlusal part of the box shape design for molar and premolar. :

All IR-FDPs were fabricated by 5 axis CAD/ CAD system (AmannGirrbach Vorarlberg, Austria). The abutments were sprayed with Shera scan spray (Shera werkstoff-technologie, Germany) then scanned using the Ceramill map 400 scanner (AmannGirrbach Vorarlberg, Austria). Data were transferred to the computer connected to the Ceramill motion 2 milling machine to analyse the two tested designs and start designing their corresponding zirconia IRFDPs in order to fabricate their restorations. The restorations were designed according to the manufacturer directions and Ceramill mind software recommendations. The restorations designs were manipulated by the software and sent to the Ceramill motion 2 milling machine. Ten samples were milled to each design. The twenty enlarged green-state partially sintered IR-FDPS were then fired using a special furnace (Ceramill therm furnace, Vorarlberg, Austria).

\section{Surface treatment of zirconia IR-FDPs:}

Before the surface treatment all IR-FDP were cleaned with $70 \%$ ethyl alcohol in an ultrasonic cleaning device for 10 minutes (Silfradent, S. SOFIA, FORL, ITALY). They were then rinsed thoroughly with water spray and dried with oil-free compressed air. Subgroups Box (AA) \& Tube (AA) were treated mechanically using $50 \mu \mathrm{m}$ Aluminium oxide particles (A12O3) applied perpendicular to the surface, using micro- blaster (Bio-art Micro Jato, Bio-art Equipamentos Odontologicos LTDA, Brazil). The specimens were mounted in a metallic holder at $10 \mathrm{~mm}$ between the surface of the sample and the micro blaster tip. Air-abrasion was performed for 10 seconds for each surface, with 2 bar pressure ${ }^{(14)}$.

\section{MDP Primer surface treatment:}

For subgroups Box (MDP) and Tube (MDP), the internal surfaces were coated with Z-PRIME plus (BISCO, Inc, Schaumburg. USA), then dried with an air syringe for 3-5 seconds according to manufacturer's instructions

\section{Abutments conditioning:}

Enamel portions of the preparations were etched for 30 seconds and the dentin surfaces of the teeth for 15 seconds with $37 \%$ phosphoric acid (Total Etch, Ivoclar Vivadent AG). Following this, all etchant gel was removed with water spray for 5 seconds. Excess moisture was removed leaving the dentin surface with a slightly glossy wet appearance. Two separate coats of the All-bond universal (BISCO, Inc, Schaumburg. USA) applied on internal surfaces by rubbing the micro brush for 10-15 second per coat. Then according to manufacturer instructions air drying using the air syringe for 10 second to allow the excess solvent to evaporate. Light curing for 10 seconds. Auto mix Duo link universal resin cement (BISCO, Inc, Schaumburg. USA) dispensed directly into the cavity covering all surfaces and on the inner surfaces of the restoration. Then each restoration was seated in place first with adequate finger pressure and the excess cement was removed immediately using a scaler. Then immediately placed under a loading device of $5 \mathrm{Kg}$ for 1 minute and light curing for 40 seconds was performed.

All specimens are subjected to thermo-mechanical cyclic loading via cyclic load multimodal ROBOTA chewing simulator integrated with thermocyclic protocol operated on servo-motor. A weight of $5 \mathrm{~kg}$, which is comparable to $49 \mathrm{~N}$. The test was repeated 37500 times accompanied with 300 thermal cycles $\left(5^{\circ}-55^{\circ}\right.$ celcius) with dwell time 25 seconds ${ }^{(15)}$.

\section{Fracture resistance evaluation:}

These tests were performed using an universal fracture testing machine (Bluehill Lite Software 
from Instron ${ }^{\circledR}$ ) all samples were individually mounted on a computer controlled materials testing machine (Model 3345; Instron Industrial Products, Norwood, MA, USA) with a loadcell of $5 \mathrm{kN}$ and data were recorded using computer software (Instron ${ }^{\circledR}$ Bluehill Lite Software). Samples were secured to the lower fixed compartment of testing machine by tightening screws. Fracture test was done by compressive mode of load applied occlusally using a metallic rod with spherical tip (5.8 $\mathrm{mm}$ diameter) attached to the upper movable compartment of testing machine traveling at cross-head speed of $1 \mathrm{~mm} / \mathrm{min}$ with tin foil sheet in-between to achieve homogenous stress distribution and minimization of the transmission of local force peaks. The load at failure manifested by an audible crack and confirmed by a sharp drop at load-deflection curve recorded using computer software (Bluehill Lite Software Instron ${ }^{\circledR}$ Instruments). The load required to fracture was recorded in Newton.

\section{RESULTS \& STATISTICAL TESTS}

An ANOVA test was used to compare between different designs and surface treatments. The significance level was set to $\mathrm{p} \leq 0.05$.

Regarding the effect of surface treatment on the fracture resistance, For tube design, there was no statistically significant difference between air abrasion and MDP as $p=0.57$. For box design, there was no statistically significant difference between air abrasion and MDP as $p=0.71$, although air abrasion showed higher fracture resistance than MDP, (Table 1).

Regards the Effect of design on fracture resistance,for Air Abrasion, there was statistically significant difference for air abrasion between tube and box design as $p=0.01$. For MDP, there was statistically significant difference for MDP between tube and box design as $p=0.0002$. Thus Box design showed higher fracture resistance than tube design for both surface treatments (Table 1).
TABLE (1) Mean values SDs for both groups and sub groups

\begin{tabular}{|c|c|c|c|c|c|}
\hline \multicolumn{2}{|c|}{ Variables } & Mean & $S D( \pm)$ & $p$ value & Significance \\
\hline \multirow{2}{*}{ Box } & $\mathrm{AA}$ & 1059.00 & 210.06 & \multirow{2}{*}{0.71} & \multirow{2}{*}{$N S$} \\
\hline & MDP & 1015.16 & 79.56 & & \\
\hline \multirow{2}{*}{ Tube } & AA & 605.72 & 129.94 & \multirow{2}{*}{0.57} & \multirow{2}{*}{$N S$} \\
\hline & MDP & 559.01 & 82.84 & & \\
\hline \multirow{2}{*}{ AA } & Box & 1059.00 & 210.06 & \multirow{2}{*}{0.01} & \\
\hline & tube & 605.72 & 129.94 & & \\
\hline \multirow{2}{*}{ MDP } & Box & 1015.16 & 79.56 & \multirow[b]{2}{*}{0.0002} & \\
\hline & tube & 605.72 & 129.94 & & \\
\hline
\end{tabular}

Regards the fracture pattern fracture in the connector area showed the highest incidence (60\%) followed by abutment + inlay $(20 \%)$, abutment (15\%) and inlay fracture showed the least (5\%).

$S=$ significant,$N S=$ non significant

\section{DISCUSSION}

In this study, extracted human teeth were used as abutments because their modulus of elasticity, bonding characteristics and strength are closer to the clinical situation than those of metal and ivory abutments. ${ }^{(16-21)}$. In order to minimize the influence of size and shape variations on the experimental results, the teeth were selected with according to their mesio-distal and bucco-lingual dimensions, straight root shape.

To imitate physiological tooth mobility, all roots of the selected teeth which were covered with an artificial periodontal membrane to simulate the movement of a tooth with a healthy periodontium. This layer made a thin layer $(0.3 \mathrm{~mm}-0.5 \mathrm{~mm}$ thickness $)$ of poly vinyl acetate (PVA) ${ }^{(22)}$, this technique used as it simple and allows to get a uniform thickness of the material all over the entire surface of the root.

In this study two designs (Box and Tube shape) were used according to the ideal guidelines for ceramic IRFDPs preparations described by 
Thompson et al ${ }^{(13)}$, and also the two tested designs are, in fact, the most used in CIR-FDPs ${ }^{(3,10.13 .23-26)}$. Firstly, each design was drawn with Auto CAD software then transferred to 5 axis CNC milling machine. $\mathrm{CNC}$ machine allows accurate preparation and same preparation for all samples.

Although, monolithic zirconia IR-FDP provide a conservative and effective modality for replacing the posterior missing molar, it needs to be cemented by resin cement. But the debonding is reported as one of the most common technical complications for zirconia-based restorations, and they still need a more reliable and long-lasting adhesion to the substrates ${ }^{(24,27,28)}$. Therefore, bonding to zirconiabased ceramics remains a challenge because of its nonreactive surface which requires different techniques than the conventional ones used for preparing silica-based ceramics, i.e. hydrofluoric acid etching and silane coupling agent. There is a wide range of possibilities concerning this issue and alternative methods have become a subject of great interest in trying to optimize zirconia. so that the restoration and tooth cavity surfaces conditioning before adhesive cementation procedures is necessary to avoid mechanical and biological complications $^{(1,29)}$.

Air-abrasion, by means of aluminum oxide particles of the zirconia intaglio surface is the most acceptable mechanical treatment since it cleans the surface and makes it a more reactive surface (microretentions and higher surface free energy), and it increases the surface area available for bonding ${ }^{(30,31)}$ . In addition, the damaging effect of air-abrasion particles on the mechanical behavior of Y-TZP materials has been reported, that researchers have recommended that the zirconia ceramic should be air-abraded at low pressure with small particle sizes of aluminum oxide ${ }^{(32)}$. In this current study, the zirconia surface was air abraded at a low pressure of $2 \mathrm{bar}$, using a powder with a particle size of $50 \mu \mathrm{m}$ to avoid surface damage ${ }^{(32)}$. To standardize the effects of the air abrasion, a custom made devise was fabricated to hold the IR-FDPs during applying the air abrasion, the devise holds the samples at constant $10 \mathrm{~mm}$ and perpendicular to the zirconia surface $^{(7,33)}$.

Also, different researches show durable adhesion to zirconia ceramic surface can be acquired by using "chemical conditioning" primers containing a phosphate-based functional monomer, especially $10-$ MDP ${ }^{(14,34-36)}$. Therefore in the present study, these two types of surface treatment for zirconia was used one mechanical (air abrasion) and one chemical method (MDP zirconia primer).

Number of cycles varies between studies range from $104-2.5^{\times} 10^{6}$ cycle, it is very popular, with general agreement that it is the most representative of the clinical situation rather than static test (15). In this study thermo-mechanical cyclic loading (TMCL) was done for 37500 cycles, $49 \mathrm{~N}, 1.6 \mathrm{~Hz}$ represent three clinical months ${ }^{(15)}$.

Due to lacking data concerning the fracture resistance of IR-FDPs the main target in the present study was directed towards evaluating the effect of design and surface treatments on the fracture resistance of monolithic zirconia IR-FDPs after thermo-mechanical loading. High fracture strength is one of the most significant prerequisites for the long-term success of all ceramic restorations.

The Box design showed higher fracture resistance than Tube design for both surface treatments. Regards the percentage of incidence of fracture at different areas of the restoration Regardless of different designs and surface treatment used, fracture in the connector area showed the highest incidence $(60 \%)$ followed by abutment + inlay $(20 \%)$, abutment (15\%) and inlay fracture showed the least (5\%).

This may be attributed to the fact that the Box design may have provided greater surface area to resist the forces than Tube design, The inlay design had an additional $2 \mathrm{~mm}$ wall of its proximal box when compared to the Tube-shaped design ${ }^{(37)}$. 
This was in agreement with Mohsen et $\mathrm{al}^{(23)}$ who evaluated the fracture resistance of three different zirconia IRFDP designs; inlay-shaped (occlusoproximal inlay + proximal box), tube-shaped (occluso-proximal inlay), and proximal box-shaped preparations. and the results indicated that IRFDPs with inlay-shaped retainers showed the highest fracture resistance values, followed by IRFDPs with tube-shaped retainers and finally IRFDPs with proximal box-shaped retainers, which recorded the lowest fracture resistance values ${ }^{(23)}$.

Reviewing the literature, Korber and Ludwig ${ }^{(38)}$ summarized that, posterior FPDs must be strong enough to withstand a load of $500 \mathrm{~N}$. They added that the highest bite force was found in the first molar region. At the same time, Hikada et al ${ }^{(39)}$ assumed maximum mastication forces of about $500 \mathrm{~N}$ in the posterior chewing areas. The fracture resistance of zirconia-based CIRFPDs recorded by all the tested groups in this research exhibited mean values ranging between $(559 \pm 82 \mathrm{~N}$ and $1059 \pm 210 \mathrm{~N})$. These results showed that, the fracture resistance of zirconia-based IRFDPs with different tested designs was greater than the maximum mastication forces. Therefore, these restorations may be strong enough for clinical applications.

This study was completed in-vitro which is considered as one of the limitations, where in-vivo study reveals better for clinical evaluation, another limitation presented in this study is that the number of thermo-mechanical cycles needs to be increased to simulate more time of clinical function.

\section{CONCLUSIONS}

Based on the findings of this in vitro study, the following conclusions can be drawn:

1. Inlay retained fixed dental prosthesis IR-FDPs with box shape provide more fracture resistance than with tube shape.

2. The air abrasion surface treatment shows similar fracture strength with the MDP primer surface treatment.

\section{REFERENCES}

1. Monaco, C., P. Cardelli, and M. Özcan, Inlay-retained zirconia fixed dental prostheses: modified designs for a completely adhesive approach. Journal of the Canadian Dental Association, 2011. 77(4): p. 237.

2. Xie, Q., L.V. Lassila, and P.K. Vallittu, Comparison of load-bearing capacity of direct resin-bonded fiber-reinforced composite FPDs with four framework designs. Journal of Dentistry, 2007. 35(7): p. 578-582.

3. Kiliçarslan, M.A., et al., In vitro fracture resistance of posterior metal-ceramic and all-ceramic inlay-retained resinbonded fixed partial dentures. The Journal of Prosthetic Dentistry, 2004. 92(4): p. 365-370.

4. Poticny, D.J. and J. Klim, CAD/CAM in-office technology: innovations after 25 years for predictable, esthetic outcomes. The Journal of the American Dental Association, 2010. 141: p. 5S-9S.

5. Griffin Jr, J.D., Tooth in a bag: same-day monolithic zirconia crown. Dentistry today, 2013. 32: p. 124,126.

6. Sailer, I., et al., Five-year clinical results of zirconia frameworks for posterior fixed partial dentures. International Journal of Prosthodontics, 2007. 20(4).

7. Sharafeddin, F. and S. Shoale, Effects of Universal and Conventional MDP Primers on the Shear Bond Strength of Zirconia Ceramic and Nanofilled Composite Resin. Journal of Dentistry, 2018. 19(1): p. 48.

8. Abduo, J., K. Lyons, and M. Swain, Fit of zirconia fixed partial denture: a systematic review. Journal of Oral Rehabilitation, 2010. 37(11): p. 866-876.

9. Abdullah, S., H. Mohammed, and K. Thayer, Factors in the failure of cemented full crowns. Dental journal, 1974. 40(11): p. 721

10. Gumus, H.S., N.T. Polat, and G. Yildirim, Evaluation of fracture resistance of inlay-retained fixed partial dentures fabricated with different monolithic zirconia materials. The Journal of Prosthetic Dentistry, 2018. 119(6): p. 959-964.

11. Monaco, C., et al., Marginal adaptation of 1 fiber-reinforced composite and 2 all-ceramic inlay fixed partial denture systems. Int J Prosthodont, 2006. 19(4): p. 373-82.

12. Kolbeck, C., et al., In vitro examination of the fracture strength of 3 different fiber-reinforced composite and 1 all-ceramic posterior inlay fixed partial denture systems. J Prosthodont, 2002. 11(4): p. 248-53.

13. Thompson, M.C., K.M. Thompson, and M. Swain, The all-ceramic, inlay supported fixed partial denture. Part 1. Ceramic inlay preparation design: a literature review. Aust Dent J, 2010. 55(2): p. 120-7; quiz 231. 
14. Kim, J., et al., Effects of multipurpose, universal adhesives on resin bonding to zirconia ceramic. Operative dentistry, 2015. 40(1): p. 55-62.

15. Nawafleh, N., et al., Lithium Disilicate Restorations Fatigue Testing Parameters: A Systematic Review. Journal of Prosthodontics, 2016. 25(2): p. 116-126.

16. Mahmood, D.J.H., E.H. Linderoth, and P. Vult Von Steyern, The influence of support properties and complexity on fracture strength and fracture mode of all-ceramic fixed dental prostheses. Acta Odontologica Scandinavica, 2011. 69(4): p. 229-237.

17. Rosentritt, M., et al., Influence of stress simulation parameters on the fracture strength of all-ceramic fixed-partial dentures. Dental Materials, 2006. 22(2): p. 176-182.

18. Rosentritt, M., et al., Influence of resilient support of abutment teeth on fracture resistance of all-ceramic fixed partial dentures: an in vitro study. International Journal of Prosthodontics, 2011.24(5).

19. Rosentritt, M., et al., In vitro fracture force and marginal adaptation of ceramic crowns fixed on natural and artificial teeth. International Journal of Prosthodontics, 2000. 13(5).

20. Scherrer, S. and W. De Rijk, The fracture resistance of all-ceramic crowns on supporting structures with different elastic moduli. International Journal of Prosthodontics, 1993. 6(5).

21. Yucel, M.T., et al., Influence of the supporting die structures on the fracture strength of all-ceramic materials. Clinical oral investigations, 2012. 16(4): p. 1105-1110.

22. Rathi, A., et al., Effect of different periodontal ligament simulating materials on the incidence of dentinal cracks during root canal preparation. Journal of dental research, dental clinics, dental prospects, 2018. 12(3): p. 196.

23. Mohsen, C.A., Fracture resistance of three ceramic inlayretained fixed partial denture designs. An in vitro comparative study. J Prosthodont, 2010. 19(7): p. 531-5.

24. Ohlmann, B., et al., All-ceramic inlay-retained fixed partial dentures: preliminary results from a clinical study. Journal of Dentistry, 2008. 36(9): p. 692-696.

25. Wolfart, S., et al., Fracture strength of all-ceramic posterior inlay-retained fixed partial dentures. Dental Materials, 2007. 23(12): p. 1513-1520.

26. Behr, M., et al., In-vitro study of fracture strength and marginal adaption of fibre-reinforced adhesive fixed partial inlay dentures. Journal of dentistry, 1999. 27: p. 163-168.

27. İzgi, A.D., E. Kale, and Ş. Eskimez, A prospective cohort study on cast-metal slot-retained resin-bonded fixed dental prostheses in single missing first molar cases: results after up to 7.5 years. Journal of Adhesive Dentistry, 2013. 15.

28. Re, D., et al., Early bond strength to low-pressure sandblasted zirconia: evaluation of a self-adhesive cement. Eur J Esthet Dent, 2012. 7(2): p. 164-175.

29. Re, S., et al., The effect of surface treatment on the adhesion of resin cements to Y-TZP. 2008.

30. Moon, J.-E., et al., Effects of airborne-particle abrasion protocol choice on the surface characteristics of monolithic zirconia materials and the shear bond strength of resin cement. Ceramics International, 2016. 42(1): p. 1552-1562.

31. Strasser, T., et al., Roughness, surface energy, and superficial damages of CAD/CAM materials after surface treatment. Clinical oral investigations, 2018. 22(8): p. 2787 2797.

32. Su, N., et al., The effect of various sandblasting conditions on surface changes of dental zirconia and shear bond strength between zirconia core and indirect composite resin. The journal of advanced prosthodontics, 2015. 7(3): p. 214-223.

33. Shehata, W.K., et al., Effect of Sandblasting and Zirconia Primer Application on The Zirconia-Cement Shear Bond Strength (An in-vitro Study). Al-Azhar Dental Journal for Girls, 2018. 5(2): p. 187-194.

34. Magne, P., M.P. Paranhos, and L.H. Burnett Jr, New zirconia primer improves bond strength of resin-based cements. Dental Materials, 2010. 26(4): p. 345-352.

35. Zandparsa, R., et al., An in vitro comparison of shear bond strength of zirconia to enamel using different surface treatments. Journal of Prosthodontics, 2014. 23(2): p. 117-123.

36. Shin, Y.J., et al., Evaluation of the shear bond strength of resin cement to Y-TZP ceramic after different surface treatments. Scanning: The Journal of Scanning Microscopies, 2014. 36(5): p. 479-486.

37. Song, H.-Y., et al., Effects of two preparation designs and pontic distance on bending and fracture strength of fiberreinforced composite inlay fixed partial dentures. The Journal of prosthetic dentistry, 2003. 90(4): p. 347-353.

38. Korber, K., Maximale Kaukraft als Berechnungsfaktor zahntechnischer Konstruktionen. Dent Lab, 1983. 31: p. 55-60.

39. Hidaka, O., et al., Influence of clenching intensity on bite force balance, occlusal contact area, and average bite pressure. Journal of Dental Research, 1999. 78(7): p. 13361344. 